\title{
Laterality Biases to Chimeric Faces in Asperger Syndrome: What is 'Right' About Face-Processing?
}

\author{
Chris Ashwin, ${ }^{1,2}$ Sally Wheelwright, ${ }^{1}$ and Simon Baron-Cohen ${ }^{1}$
}

\begin{abstract}
People show a left visual field (LVF) bias for faces, i.e., involving the right hemisphere of the brain. Lesion and neuroimaging studies confirm the importance of the right-hemisphere and suggest separable neural pathways for processing facial identity vs. emotions. We investigated the hemispheric processing of faces in adults with and without Asperger syndrome (AS) using facial emotion and identity chimeric tasks. Controls showed an LVF bias in both tasks, but no perceptual bias in a non-social control task. The AS group showed an LVF bias during both tasks, however the bias was reduced in the identity condition. Further, the AS group showed an LVF bias in the non-social condition. These results show a differential pattern of hemispheric processing of faces in AS.
\end{abstract}

KEY WORDS: Asperger syndrome; autism; face-processing; social cognition; brain laterality; emotional expression.

\section{INTRODUCTION}

In the late nineteenth century Broca (1865) and Wernicke (1874) both made observations that patients with lesions in the left hemisphere of their brains had lost certain language abilities. Subsequently it was also noted that patients with right hemisphere damage had deficits in spatial perception (Jackson, 1915; Riddoch, 1935; Patterson \& Zangwill, 1944). Since the time of these reports cognitive neuroscience has been interested in the lateralisation of cognitive functioning. It is now generally accepted that the two hemispheres of the brain are specialised for different kinds of information processing, with the left hemisphere relatively specialised for language and the right hemisphere for visuospatial cognition

\footnotetext{
${ }^{1}$ Departments of Psychology and Psychiatry, Autism Research Centre, University of Cambridge, Cambridge, UK

${ }^{2}$ Correspondence should be addressed to Chris Ashwin, Departments of Psychology and Psychiatry, Autism Research Centre, University of Cambridge, Douglas House, 18b Trumpington Rd, Cambridge CB2 2AH, UK. Tel: + 44 (01223) 746030; Fax: + 44 (01223) 746033; e-mail: ca235@cam.ac.uk
}

(Sperry, Gazzaniga \& Bogen, 1969; Hellige, 1993; Davidson \& Hugdahl, 1995).

Among the visuospatial stimuli the right hemisphere is thought to govern are faces. This idea stems from a number of studies with normal populations and brain-damaged individuals. For example, neuroimaging studies have consistently shown activation of the fusiform gyrus to faces, usually on the right side (Kanwisher, McDermott \& Chun, 1997; McCarthy et al., 1997; Haxby et al., 1999). In prosopagnosia, a disorder of face perception (most commonly identity), lesions are almost always located in the right hemisphere (Young, 1998). It has also been shown that patients with right temporal cortex excisions, but not left, have face-memory impairments (Milner, Taylor \& Sperry, 1968; Crane \& Milner, 2002). Emotion recognition studies have found that people with right-hemisphere damage are impaired relative to controls and people with left-hemisphere damage (Bowers, Bauer, Coslett \& Heilman, 1985; Borod et al., 1998; Adolphs, Damasio, Tranel, Cooper \& Damasio, 2000). In general, it appears the right 
hemisphere contains systems for social communication, which in many ways may be complementary to the left hemispheres specialization for language (Bowers, Bauer \& Heilman, 1993).

Wolff (1933) was among the first to suggest that the left half of the face is judged to look more like the whole face than the right half. Since this time chimeric face tasks have been central for investigating perceptual asymmetries in face processing. Chimeric face stimuli often involve faces that have been split in a horizontal (left-right) or a vertical (up-down) orientation, with the two halves differing on a visual dimension. Gilbert and Bakan (1973) found that the "observer" shows a left visual field (LVF) in an identity face-processing task. They used a chimeric task involving judegments of identity with two composite faces being compared to an original target face. Kolb, Milner \& Taylor (1983) extended these findings in people with various brain lesions. Like the previous study, controls showed a LVF bias in the face identity chimeric task. In contrast, patients with lesions in the right temporal lobe, including the amygdala, performed at chance level and did not show a visual field bias.

Another variation of the chimeric face involves half of a face expressing an emotion and the other half being neutral. Like the chimeric face tasks involving judgments of identity, experimental findings with left-right asymmetrical stimuli with facial expressions of emotion have consistently reported a LVF bias (Burt \& Perrett, 1996; Drebing, Federman, Edington \& Terzian, 1997). This LVF bias for processing faces represents mainly right hemisphere functioning in the brain, as information from the LVF crosses at the optic chiasm and travels to the right visual cortex. When people view chimeric faces made from combining a smiling half and a neutral half of a face together, the expression presented to their LVF dominates the judegment of the emotion they identify (Campbell, 1978; Heller \& Levy, 1981). This LVF preference is thought to reflect a bias for facial emotion processing by right hemisphere systems of the brain. This is in line with the neuropsychological and neuroimaging studies showing the importance of the right hemisphere in various aspects of face processing, reviewed earlier.

Some findings however suggest that the model of hemispheric processing of facial stimuli might be more complex. Thus, two hypotheses have emerged to explain the laterality of emotion processing. The "right hemisphere" hypothesis views the right hemisphere as specialized for all types of emotions
(Christman \& Hackworth, 1993; Borod et al., 1998). The "valence" hypothesis sees the right hemisphere as specialized for negative emotions and the left hemisphere specialized for positive emotions (ReuterLorenz \& Davidson, 1981; Reuter-Lorenz, Givis \& Moscovitch, 1983).

Support for lateralised perceptual processing of emotional visual stimuli comes from a number of studies. Reuter-Lorenz and Davidson (1981) found that tachistoscopic presentation of emotional faces revealed an LVF bias for perception of sad faces and a right visual field (RVF) bias for perceiving happy faces. Davidson, Mednick, Mass, Saron and Schaffer (1987) replicated and extended this finding, and suggested that such a pattern is inconsistent with the notion that the right hemisphere alone is involved in processing all emotions. Using chimeric faces and a free-viewing administration, Levy, Heller, Banich and Burton (1983) showed that right-handed subjects judged faces as happier when the smile was on the left side of the face (with neutral on the right), when compared with the converse. Replications and extensions of these findings were reported in subsequent studies (Hoptman \& Levy, 1988; Luh, Ruekert \& Levy, 1991). Finally, using a similar paradigm in which chimeric faces showed an emotion on one side and a neutral expression on the other, Christman and Hackworth (1993) found greater accuracy in identifying the emotion when the emotion was on the left half of the chimera. Unlike the other studies, this LVF bias was obtained for both positive and negative emotions.

An influential model of face processing (Bruce \& Young, 1986) posits separate mechanisms for identity vs. emotion perception. Neuropsychological findings support this notion: evidence shows a double dissociation in patients with different lesions in deficits for identity vs. emotion perception (Bornstein, 1963; Kurucz \& Feldmar 1979; Kurucz, Feldmar \& Werner, 1979; Tranel, Damasio \& Damasio, 1988; Young, 1998). This double dissociation strongly suggests there are separate systems for identity vs. emotion processing in the brain, although both appear to be mediated more by right-hemisphere processing. Haxby and colleagues have investigated the neural mechanisms of face-processing using functional neuroimaging, and hypothesize two major visual routes in the brain for processing facial information that involve separate components for identity and emotional expression processing (Hoffman \& Haxby 2000; Haxby, Hoffman \& Gobbini, 2002): a more dorsal route, which includes the superior 
temporal sulcus (STS), more involved in processing dynamic aspects of the face, including emotional expressions; and a second more ventral route, involving more the fusiform gyrus, concerned with processing static aspects of the face like identity. Neuroimaging tasks that involve processing dynamic aspects of the face like gaze, movements of the mouth and eyes, or emotional expressions consistently activate the right STS (Bonda, Petrides, Ostry \& Evans, 1996; Allison, Puce \& McCarthy, 2000; Hoffman \& Haxby, 2000). Neuroimaging tasks that involve facial identity tend to activate the fusiform gyrus, usually on the right side (Kanwisher et al., 1997; McCarthy, Puce, Gore \& Allison, 1997; Haxby, Hoffman \& Gobbini, 2000; Haxby et al., 2002; Kanwisher, 2000). These findings not only illustrate the separation in mechanisms for processing facial emotions versus identity, but they also show that faces tend to preferentially activate the right hemisphere of the brain.

Asperger syndrome (AS) is a neurodevelopmental condition characterized by deficits in social and communicative functioning and by repetitive behaviour and interests (APA, 1994). Among the more striking characteristics of AS are difficulties in social cognition, including face and emotional expression processing (Kanner, 1943; Asperger, 1944; Baron-Cohen, Wheelwright \& Jolliffe, 1997). These difficulties often exist alongside intact performance in other areas of cognitive functioning such as intelligence and executive functioning (Baron-Cohen, Wheelwright, Stone \& Rutherford, 1999; Fine, Lumsden \& Blair, 2001). Some theories hold that deficits in social-emotional functioning are central features of AS (Kanner, 1943; Hobson, 1993; BaronCohen, 1995; Waterhouse, Fein \& Modahl, 1996; Schultz, Romanski \& Tsatsanis, 2000), although to date little is known about hemispheric processing during social cognition in autism spectrum disorders (ASD).

Various hypotheses have been proposed regarding which side of the brain might be involved in ASD. These hypotheses have at different times implicated the left hemisphere, the right hemisphere, or both hemispheres (Geschwind \& Galaburda, 1987; Klin, Volkmar, Sparrow, Cicchetti \& Rourke, 1995; Rinehart, Bradshaw, Brereton \& Tonge, 2002a). Early ideas centred on left-hemisphere dysfunction (LHD), because of the language deficits involved in autism (Tanguay, Ornitz, Forsythe \& Ritvo, 1976; Blackstock, 1978; Prior \& Bradshaw, 1979). This idea of LHD was also consistent with other behaviours seen in autism. For example, people with damage to the left hemisphere are often more literal, a feature commonly found in autism (Gillberg, 2000). The increased rate of left-handedness (or non-right handedness) in ASD has also been attributed to damage to the left hemisphere (Hauser, DeLong \& Rosman, 1975; McManus, Murray, Doyle \& BaronCohen, 1992). Another view is that ASD may be characterized by inconsistent lateral preference (Escalante-Mead, Minshew \& Sweeney, 2003). Increased left and ambiguous-handedness are of course not unique to ASD, as such patterns are also elevated in people with learning difficulties, Down"s syndrome and epilepsy (Lewin, Kohen \& Mathew, 1993; Cornish \& McManus, 1996). The findings of handedness in ASD have been questioned however by the lack of consistent measures across studies for determining hand preference (Bryson, 1990). It may also be that such handedness profiles reflect different neurodevelopmental pathways rather than a specific neurological insult (Fein, Waterhouse, Lucci, Pennington \& Humes, 1985).

The lack of consistent support for an LHD, along with the emerging models that the social deficits may have primacy over the language impairments, led to the idea of a right hemisphere dysfunction (RHD) in ASD (Ellis \& Gunter, 1999). The RHD hypothesis often refers less to "classic" autism and more to high-functioning autism (HFA) or AS, where early language impairments are absent. In people with AS, syntactic and lexical development is often intact, but the pragmatics of language is invariably impaired (Baron-Cohen, 1988; Ozonoff \& Miller, 1996; Martin \& McDonald, 2003). The behaviour of people with AS is noted to be similar to people with damage to the right hemisphere (Gunter, Ghaziuddin \& Ellis, 2002). The right hemisphere is thought to be essential for non-verbal aspects of communication like gesture and emotion recognition, which are impaired in people with AS, further suggesting the right hemisphere may be involved (Ellis \& Gunter, 1999). Neurobehavioural evidence supports a right hemisphere deficit model in AS (Ellis, Ellis, Fraser \& Deb, 1994), however evidence of both left and right dysfunction exist (Rinehart, Bradshaw, Brereton \& Tonge, 2002b).

Given the importance of social deficits to AS and the conflicting results, it is of interest to test if faces are processed normally in the right hemisphere, via the LVF, as a further test of the lateralization of cognitive functions. Such effects might not be specific to emotion processing, so in the experiment reported below we include other types of non-social 
asymmetric stimuli. Specifically, we include asymmetric arrays of stars, which have no known dedicated neural systems. The present study had the following aims: (1) To test an LVF bias for faces in controls using both an identity and an emotion chimeric task; (2) To investigate any differences in people with AS for visual field biases during both face identity and emotional expression chimeric face tasks.

We predicted the controls would show a bias for the left visual field choice in both the identity and emotion expression chimeric face tasks, and would perform at chance levels for the non-social star condition. We predicted the participants with AS might show an atypical profile on either the identity or the emotion face-processing task, or both; and that this might also be revealed in a non-social control condition, if there was a right hemisphere abnormality.

\section{EXPERIMENT 1}

We investigated right-hemisphere processing during social cognition in people with and without AS using a chimeric face task involving emotional expressions. The chimeric face experiment involved two social conditions (happy and angry facial expressions), and one non-social control condition (pictures of stars).

\section{METHODS}

\section{Participants}

We recruited 16 male adult control participants (mean age $\pm S D, 28.3 \pm 7.0$; full-scale IQ, $117.7 \pm$ 16.3) with no history of psychiatric conditions from the general population through advertisements. We also recruited 16 adult male participants (mean age $\pm S D$, $26.8 \pm 7.8$; full-scale IQ, $118.4 \pm 17.6)$ who had a formal diagnosis of AS according to international criteria (APA, 1994). Every participant except for one in each group completed an assessment of intelligence (Wechsler, 1999), and all of the participants with AS except for one also completed the Autism-Spectrum Quotient (AQ) (Baron-Cohen, Wheelwright, Skinner, Martin \& Clubley, 2001). The AQ scores of the participants with AS $(n=15$, mean AQ score $=33.7$, $S D=7.7,73.3 \%$ scoring $32+$ ) matched very closely to those found previously in Baron-Cohen et al. (2001) $(n=58$, mean AQ score $=35.8, S D=6.5,80 \%$ scoring $32+$ ).

\section{Materials}

To create the stimuli we chose pictures of males and females expressing the emotions of anger and happiness from a standard set of facial expression pictures (Lundqvist, Flykt \& Ohman, 1998). To ensure each picture expressed the appropriate emotion, we showed 10 judges the pictures and asked them to pick which emotion each picture portrayed from a list of the basic emotions (anger, disgust, fear, surprise, happy, sad, and neutral), placed on the desk in front of them. We then chose five male and five female pictures for each of the angry and happy faces that all ten judges showed agreement on, and a neutral picture corresponding to each of them. This made a total of ten different emotional photographs depicting happy (five male and five female), 10 neutral expression photographs corresponding to the happy pictures (five male and five female), 10 different emotional photographs for angry (five male and five female), and 10 neutral expression pictures corresponding to the angry pictures (five male and five female).

Using Paint Shop $\operatorname{Pro}^{\odot}$ we split each of the pictures down the middle of the faces, and paired the left side of each emotional face with the right side of the neutral face. We also paired the right side of each emotional face with the left side of the neutral face. This created two chimeric faces for each picture, one with the left of the emotional face paired with the right side of the neutral face, and one with the right side of the emotional face paired with the left side of the neutral face. We mirrored each picture horizontally to create a new version of each face that was identical, but with the emotional and neutral sides reversed. One chimeric face picture and its mirrorimage appeared in each display, one above and one below the other. Two versions of each display were created, with the left-sided chimera appearing at the top of the display in one version and at the bottom in the other version to counterbalance the location of the left-chimeric emotional display. This made a total of 40 happy chimeric face displays, and 40 angry chimeric face displays (see Fig. 1a).

We took pictures of stars in space from the Internet, and copied and pasted more stars on one side than the other to create chimeric star pictures. Each picture was then mirrored horizontally to create another identical copy, but with the majority of stars now on the opposite side. One chimeric star picture and its mirror image appeared in each display, one above and one below the other (see Fig. 1a). There 

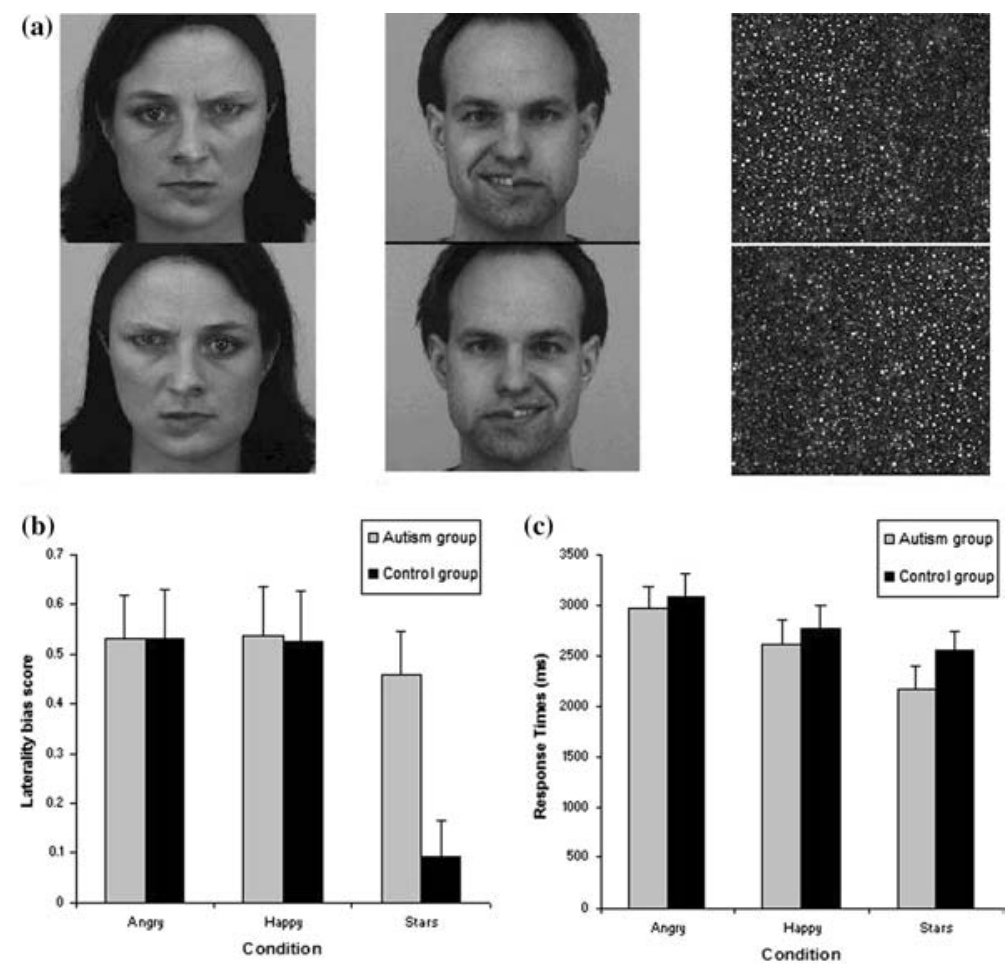

Fig. 1. (a) Examples of angry, happy, and non-social star chimeric pictures used for Experiment 1 Photographs adapted with permission from CD ROM "The Korolinska Directed Emotional Faces-KDEF" (Lundqvist et al. 1998). (b) Mean laterality bias scores for the control group (dark bar) and AS group (grey bar) for each condition. (c) Mean response latencies for the control group (dark bar) and AS group (grey bar) for each condition.

were two display versions for each star picture, with the left-sided chimera appearing at the top of the display in one version and at the bottom in the other version. Ten different chimeric star displays were created, each with two versions, to create 20 in total. These were repeated twice during the experiment to make 40 trials, matching the other two conditions.

\section{Experimental Design}

The computer-generated free-viewing chimeric face tasks were carried out in a specially equipped and dimly lit experimental room. Participants were first given written instructions on the task, which were then explained verbally to them. Instructions also appeared on the computer screen before each of the three conditions to ensure participants knew which task they were performing. In the angry chimeric face condition they were shown a series of angry chimeric displays and asked to choose which one they thought looked angrier. Participants were instructed to press one button if they thought the top picture in the display was the most appropriate answer, and another button if they chose the bottom picture. For the happy chimeric face condition, they were shown the happy chimeric displays asked which one they thought looked happier. In the star chimeric condition they were shown the star chimeric displays and asked which one they thought had more stars. They were told to guess if they needed to on any of the trials, and not to count the stars in the star chimera condition. The buttons were reversed for half the participants. Eight practice trials were done first to ensure the participants understood the task.

The experiment was run on a DELL Inspiron 7500 laptop computer using DMDX (Forster \& Forster, 2003) to present the pictures and record the responses. Participants made their responses on a specially constructed response box connected to the computer. The experiment was projected to a 20 -inch monitor situated approximately $60 \mathrm{~cm}$ in front of the participants at eye level. One trial consisted of a fixation cross that was shown for 1000 milliseconds and followed by a picture. Each trial was terminated by the participants response or a maximum of 6 seconds, whichever came first. The experiment was done in three blocks, with 40 trials in each block. 


\section{Statistical Design}

The measure we were primarily interested in was the laterality bias score, which is a measure of the advantage of visual fields. Laterality bias scores were calculated for each subject for each condition by taking the number of positive identifications of leftbiased chimera's minus the number of positive identifications of right-biased chimera's divided by the total number of chimera's. These scores range from -1 to +1 , with positive scores representing an LVF advantage. We were also interested in reaction time (RT) scores by participants.

A general linear model ANOVA with repeated measures was performed on the laterality bias scores, with Chimera Condition (angry faces vs. happy faces vs. stars) as the within-subject factor, and Group (controls vs. AS) as the between-subject factor. A general linear model ANOVA with repeated measures was also performed on the RT measures, with Chimera Condition (angry faces vs. happy faces vs. stars) as the within-subject factor, and Group (controls vs. AS) as the between-subject factor. Post-hoc pairwise comparisons and paired-samples $t$-tests were done where appropriate. One-sample $t$-tests were done for the data of both groups on each condition to test whether their scores were significantly greater than zero (i.e., chance levels).

\section{RESULTS}

Independent-samples $t$-tests showed the two groups did not differ from each other on age, $t(30)=0.571, n s$, or full-scale IQ, $t(28)=0.108, n s$.

Table I shows the laterality bias scores and RT"s of the groups for the three conditions.

The analysis of the laterality bias scores showed there was no main effect of group, $F(1,30)=1.54$, $n s$. However, there was a significant main effect of chimera condition, $F(2,29)=7.53, p=.002$. Pairwise comparisons revealed the laterality bias score for the star condition was significantly lower than both the angry and happy chimeric conditions, with no difference between the angry and happy scores (corrected for multiple comparisons with LSD). Most importantly, there was an interaction between chimera condition and group, $F(2,29)=3.68$, $p<.05$. Post-hoc paired-samples $t$-tests for the AS group revealed there was no significant difference between the laterality bias scores for any of the three conditions ( $p<0.05$ for every comparison). Paired-
Table I. Characteristics and Mean Scores of the Participant Groups for Experiment 1.

\begin{tabular}{lcr}
\hline & \multicolumn{2}{l}{ Group } \\
\cline { 2 - 3 } Characteristics and tasks & \multicolumn{1}{l}{ AS } & \multicolumn{1}{l}{ Controls } \\
\hline Age (years, mean \pm SD) & $26.8 \pm 7.8$ & $28.3 \pm 7.0$ \\
IQ (mean FSIQ \pm SD) & $118.4 \pm 17.6$ & $117.7 \pm 16.3$ \\
Gender (male/female) & $16 / 0$ & $16 / 0$ \\
Handedness (right/left) & $14 / 2$ & $14 / 2$ \\
Angry chimeric face task & & \\
$\quad$ Mean laterality bias $\pm S D$ & $.53 \pm .34$ & $.53 \pm .39$ \\
$\quad$ Mean RT \pm SD & $2901.8 \pm 858.9$ & $3078.8 \pm 915.2$ \\
Happy chimeric face task & & \\
$\quad$ Mean laterality bias \pm SD & $.54 \pm .38$ & $.53 \pm .40$ \\
$\quad$ Mean RT \pm SD & $2569.5 \pm 979.8$ & $2775.6 \pm 901.5$ \\
Star chimeric picture task & & \\
$\quad$ Mean laterality bias \pm SD & $.46 \pm .35$ & $.09 \pm .29$ \\
$\quad$ Mean RT \pm SD & $2148.8 \pm 889.5$ & $2562.2 \pm 770.3$ \\
\hline
\end{tabular}

samples $t$-tests done on the control group data revealed significant differences between the star chimeric condition and both the angry, $t(15)=3.92$, $p<.01$, and the happy chimera conditions, $t(15)=4.05, p<.01$ (see Fig. 1b). There was no difference in laterality bias score between the angry and the happy chimeric conditions for the control group, $t(15)=0.11, n s$.

One-sample $t$-tests on the data for the AS group showed they were performing above chance on the angry, $t(15)=6.36, \quad p<.001$, happy faces, $t(15)=5.70, \quad p<.001, \quad$ and star conditions, $t(15)=5.25, p<.001$. The control group were performing above chance on the angry, $t(15)=5.49$, $p<.001$, and happy chimeric face conditions, $t(15)=5.32, p<.001$, but were below chance for the non-social star condition, $t(15)=1.26, n s$.

The analysis of the RTs showed a main effect of chimera condition, $F(2,29)=14.23, p<.001$. Posthoc pairwise comparisons $(p<.05$ with least significant difference adjustments) revealed that RTs for the angry chimera condition were longer than the happy chimera condition, which were longer than the stars condition (see Fig. 1c). There was no effect of group, $F(1,30)=0.84, n s$, and no interaction between chimeric condition and group, $F(1,30)=0.73$, ns.

\section{DISCUSSION}

The laterality bias results revealed both the control and AS groups showed an LVF bias for the 
emotional chimeric faces. This finding of normal hemisphere asymmetry during judgements of chimera faces composed of basic emotional expressions is consistent with previous findings of at least some intact recognition of basic emotions in HFA/AS (Adolphs, Sears \& Piven, 2000; Baron-Cohen et al., 1997; Golan, Baron-Cohen \& Hill, in press). In the non-social condition the control group did not show a perceptual bias for either visual field, but the group with AS still displayed an LVF bias. The results suggest that AS involves normal hemispheric asymmetries during the processing of basic emotional expressions. However, the AS group was atypical in showing an LVF bias during the non-social condition. This may reflect an over-dependence on the right hemisphere, or perhaps a dysfunction of the left hemisphere.

The analysis of RTs revealed a main effect of chimeric condition, but no main effect or interactions involving group. Both groups showed longer RTs in the angry chimeric condition, followed by the happy chimeric condition, and then the star condition. This is consistent with a stroop interference effect to negative/threatening stimuli compared to happy or neutral stimuli (Williams, Mathews \& Macleod, 1996). Findings from emotional stroop paradigms with threatening faces and stimuli show an interference effect on behaviour, resulting in slower responses (C. Ashwin, S. Wheelwright \& S. Baron-Cohen, unpublished data; van Honk et al., 1998, 2000). This is interesting since the stroop effect was apparent in our paradigm even with facial expressions presented in only one visual field. In Experiment 2 below, we tested whether we would find the same laterality bias effects in the control and the AS groups in a chimeric task involving face identity judgements.

\section{EXPERIMENT 2}

\section{METHODS}

\section{Participants}

All of the participants who took part in Experiment 1 also participated in Experiment 2, except for one in each of the groups. Therefore, there were 15 adult male participants with AS (mean age $\pm S D$, $26.7 \pm 8.2$; full-scale IQ, $117.4 \pm 20.0)$ and 15 adult male controls (mean age $\pm S D, 28.3 \pm 7.0$; fullscale IQ, $118.0 \pm 14.9)$. One of the participants with
AS did not complete the IQ, and another AS participant did not complete the AQ. The AQ scores of the participants with AS $(n=14$, mean AQ score $=33.8, S D=8.0,71.4 \%$ scoring $32+$ ) matched very closely to those found in Baron-Cohen et al. (2001) $(n=58$, mean AQ score $=35.8$, $S D=6.5,80 \%$ scoring $32+$ ).

\section{Material}

We chose pictures of males and females with neutral pictures from the same standard set of facial pictures as had been used in Experiment 1 (Lundqvist et al., 1998). We showed the pictures to 10 judges and had them label each from a choice of the basic emotions including neutral. From the pictures that all 10 judges rated as neutral, we chose 10 male and 10 female photographs. Using Paint Shop Pro ${ }^{\odot}$ we split each of the pictures down the middle of the faces, to create a left side and a right side of each face. We then horizontally mirrored each of these "half faces", to create an identical version with reversed horizontal orientation, and then we joined each half up to its mirrored pair. This created two new versions of each original face, a left-left composite and a right-right composite. We then used the original neutral faces and the composite faces to make chimeric displays, similar to the stimuli used by Kolb et al. (1983) (see Fig. 2a).

Each chimera picture had an original neutral face, and a left-left version and a right-right version for comparison. Four versions of each set of chimeric stimuli were created, to counterbalance the positions of all the faces vertically and horizontally. The original face appeared in the top in one version, and the bottom in another. We also counterbalanced the position of each composite picture to appear on the left in one version and the right in another. Since we used 10 male and 10 female neutral pictures, this produced 40 female chimeric pictures and 40 male chimeric pictures in total. Since eight were used as practice trials, this left a total of 72 pictures in the experiment.

\section{Experimental Design}

The experiment procedure for this task was very similar to Experiment 1. For this task participants were asked to choose which of the two composite faces they thought looked more like the original face. They were instructed to press the left button for the left composite and the right button for the right composite, and they had a maximum of 10 seconds to 

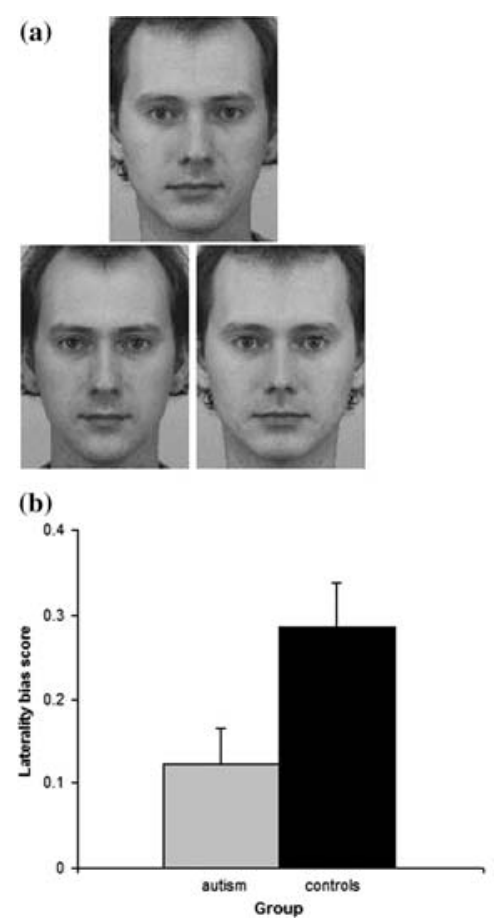

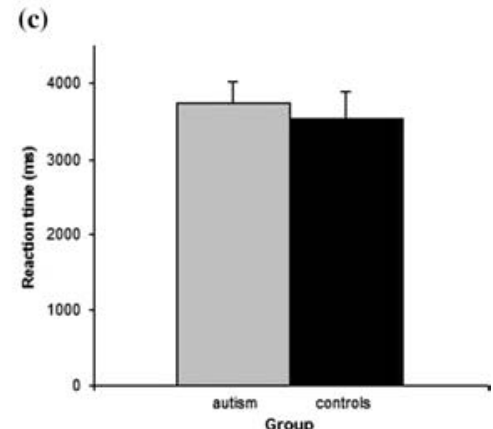

Fig. 2. (a) Examples of identity chimeric faces used for Experiment 2 Photographs adapted with permission from CD ROM "The Korolinska Directed Emotional Faces-KDEF" (Lundqvist et al. 1998). (b) Mean laterality bias scores for the control group (dark bar) and AS group (grey bar) for Experiment 2. (c) Mean response latencies for the control group (dark bar) and AS group (grey bar).

respond before the trial moved on. The experiment was randomised and had three blocks, with 24 trials in each block.

\section{Statistical Design}

Once again the measure we were primarily interested in was the laterality bias score, which is a measure of the advantage of the visual fields. We also analysed the RTs for each of the participants for both groups. Independent sample $t$-tests were carried out with the laterality scores and the RTs to investigate any group differences. One-sample $t$-tests were done on the data of both groups to test whether their laterality bias scores were significantly greater than zero (i.e., chance levels).

\section{RESULTS}

Independent-sample $t$-tests showed the two groups did not differ from each other on age, $t(28)=0.602, n s$, or full-scale IQ, $t(27)=0.088, n s$.

Table II shows the laterality bias scores and RTs of the two groups for the face identity chimeric task.
Table II. Characteristics and Mean Scores of the Participant Groups for Experiment Two.

\begin{tabular}{lcc}
\hline & \multicolumn{2}{l}{ Group } \\
\cline { 2 - 3 } Characteristics and tasks & AS & \multicolumn{1}{l}{ Controls } \\
\hline Age (years, mean $\pm \mathrm{SD}$ ) & $26.7 \pm 8.2$ & $28.3 \pm 7.0$ \\
IQ (mean FSIQ $\pm \mathrm{SD}$ ) & $117.4 \pm 20.0$ & $118.0 \pm 14.9$ \\
Gender (male/female) & $15 / 0$ & $15 / 0$ \\
Handedness (right/left) & $13 / 2$ & $13 / 2$ \\
Face identity chimeric task & & \\
$\quad$ mean laterality bias $\pm \mathrm{SD}$ & $.12 \pm .16$ & $0.29 \pm 0.20$ \\
$\quad$ mean RT $\pm \mathrm{SD}$ & $3738.3 \pm 1111.6$ & $3528.4 \pm 1383.4$ \\
\hline
\end{tabular}

Analysis of the laterality bias scores revealed a significant difference between the two groups, $t(28)=2.45, p<.05$, and looking at Fig. $2 \mathrm{~b}$ it is clear to see the laterality bias score for the control group is greater than the AS group. There was no significant difference between the groups for RTs, $t(28)=0.458, n s$, as shown in Fig. 2c.

One-sample t-tests showed that both the control group, $t(14)=5.58, p<.001$, and the AS group, $t(14)=2.87, p<.02$, were scoring significantly greater than zero (i.e., above chance levels) for the chimeric face identity task. 


\section{DISCUSSION}

The controls in this experiment showed an LVF bias in their choice of composite faces, consistent with previous research. The AS group showed an LVF bias, but the laterality bias score was significantly lower compared to the control group. The difference in hemisphere bias for face processing in the AS group is not likely to be due to the difficulty of the task, since the RTs were not different between the groups. These results suggest that during identity discrimination, people with AS do not utilise right hemisphere faceprocessing mechanisms to the same degree as controls. The decreased laterality of function may be involved in the deficits seen in people with AS with tasks involving face identity and memory.

\section{GENERAL DISCUSSION}

This study found that controls showed a LVF bias during both the emotional expression and the face identity chimeric tasks, consistent with previous studies (Kolb et al., 1983; Levy et al., 1983). The AS group showed a normal LVF bias in the emotional expression task, but in the identity task they showed a significantly reduced LVF bias compared to the controls. The AS group also showed a strong LVF bias during the non-social condition, in which the controls performed at chance levels for visual field preference. These findings imply that some hemispheric aspects of face processing may be normal in AS, but in our experiment this was not evident in all tasks and not always to the same degree as controls.

Our findings indicate a normal laterality bias for emotional faces in participants with AS, but reduced laterality bias during a task involving face identity. These results are generally consistent with results from previous studies of facial identity and emotion expression in ASD. The emotional expression chimeric task in the present study involved basic emotions, and people with HFA/AS are reported to show intact performance with basic emotions (Adolphs et al., 2000; Baron-Cohen, Spitz \& Cross, 1993; Baron-Cohen et al., 1997; Golan et al., in press; Grossman, Klin, Carter \& Volkmar, 2000; Loveland et al., 1997), although also see Hobson (1993) and Celani, Battacchi and Ardiacono (1999). A recent study involving neuroimaging and behavioural paradigms of labeling basic emotions found no difference between participants with and without ASD at the neural or behavioral level (Wang, Dapretto, Hariri, Sigman \& Bookheimer, 2004).

Our finding of a significantly reduced LVF bias in a facial identity chimeric task is consistent with previous findings. Impaired processing of facial identity in ASD was first shown by Langdell (1978), and has been replicated since then with both low and high functioning individuals (Tantam, Monaghan, Nicholson \& Stirling, 1989; De Gelder, Vroomen \& Van der Heide, 1991; Boucher \& Lewis, 1992; Davies, Bishop, Manstead \& Tantam, 1994; Boucher, Lewis, \& Collis, 1998; Klin et al., 1999), but also see Celani et al (1999) and Ozonoff, Pennington and Rogers (1990). The facial identity deficit found previously in ASD also includes delayed recognition memory (Hauck, Fein, Maltby, Waterhouse \& Feinstein, 1998). Neuroimaging studies looking at the perception of facial identities in people with and without ASD have found significant activity in the fusiform gyrus, greater on the right than the left, in controls and reduced or absent activations of the fusiform gyrus in those with ASD (Pierce, Muller, Ambrose, Allen \& Courchesne, 2001; Schultz et al., 2000). Kolb et al. (1983) used the same facial identity chimeric task and found that people with lesions of the right temporal lobe, including the amygdala, failed to show a visual field bias. The participants with AS in our study showed a significant LVF bias, however this bias was reduced compared to the control group. Our findings suggest that the right temporal lobe system in AS may involve a lack of normal bias for facial identity, although the mechanisms are not absent or lost.

The control group in our study showed no visual field bias in the non-social condition, while the participants with AS showed a strong LVF bias. This would suggest an over-dependence on the right hemisphere in AS that extends to the processing of non-social information, an effect not seen in the controls. However, it is unclear from our study whether this finding represents overgrowth of the right hemisphere or a dysfunction of the left hemisphere. The model of Geschwind and Gallaburda (1987) posits that the right hemisphere develops faster in typical males compared to females, as a function of the amount of fetal testosterone produced. The current results may be evidence for an extreme righthemisphere style of processing in AS, which is consistent with being hyper-masculinised in neurocognitive terms.

The differential results across the face-processing tasks in our study indicate hypotheses about brain 
lateralisation in AS may be more complex than often conceptualized, even within a single domain such as social cognition. Therefore, a simple encompassing left or right impairment may not be appropriate. Rinehart et al. (2002b) carried out a series of lateralisation tasks in people with ASD and found that the AS group showed evidence of RHD in some, but not all, tasks. They proposed that the key factor involved was the degree of executive function (EF) demand in certain tasks: when EF was required, participants with AS showed RH deficits. When the task did not require $\mathrm{EF}$, such as in the chimeric face task, they no longer showed evidence of RHD (Rinehart et al., 2002b). They interpreted the deficits in tasks requiring $\mathrm{EF}$ as reflecting a disruption of a fronto-striatal circuit involved in higher-order cognitive abilities, which was not evident in their chimeric face task.

This idea is not consistent with our findings, as our tasks were simple, non-verbal, required very little memory, and would therefore be expected to involve little EF. Yet we still found a differential pattern of normal and abnormal bias in hemispheric functioning in AS, depending on the task involved. We cannot rule out that EF was involved to some degree in our chimeric face tasks, or that it might be differentially involved in the two experiments. There were three faces in each picture of the identity chimeric task, compared to two in each picture of the emotional expression chimeric task. Although both tasks were simple, the added complexity in the identity task may have recruited more EF and contributed to the reduced perceptual asymmetry. Further investigations are required to test this further.

We did not find evidence for a valence effect in the two hemispheres. Recent studies have found a specific left visual field bias for faces with negative expressions, and a right visual field bias for positive emotions (Jansari, Tranel \& Adolphs, 2000; Adolphs, Jansari \& Tranel, 2001). Our results are consistent with the right hemisphere hypothesis, and do not support the valence hypothesis. However, differences in results may be due to variation in the paradigms and stimuli used. For example, recent studies finding evidence for the valence hypothesis have used stimuli composed of two whole faces, one appearing to the left of midline and the other appearing to the right (Jansari et al., 2000; Adolphs et al., 2001). This horizontal display of emotional faces differed from our vertical display, which may have contributed to the difference in results. Our study also used faces composed of neutral and emotional halves, while the previous studies by Adolphs and his colleagues used whole faces that were either neutral or emotional, with the entire face appearing in one visual field or the other. Future studies of social lateralisation processing in ASD could incorporate both types of designs to test the differential effects that various stimuli and paradigms have on performance.

The findings of an LVF bias in the emotional expression condition appears to be evidence of holistic processing of faces in AS. A recent Positron Emission Tomography (PET) study (Rossion et al., 2000) investigated activity in the bilateral fusiform gyrus using conditions involving part versus whole processing of faces. They found that the right fusiform gyrus was activated more for configural face processing, while the left fusiform gyrus was activated more for the featural face processing condition. These findings were interpreted as showing a dissociation of separate lateralised mechanisms for featural versus holistic processing of faces. The findings from our study suggest normal holistic face processing by the participants with AS in the emotional expression task, but less of a holistic style of processing during the face identity task. Further work should investigate possible differences in processing style as a factor of the type of task.

One explanation for the findings of an LVF bias for the non-social star condition in the participants with AS is that the non-social star condition was presented last for every participant. This may have produced an order effect on the results for people with AS, a condition characterized by repetitive and perseverative behaviour. We feel this is an unlikely explanation, but again, future studies should counterbalance the order of social and non-social conditions to test the results are not due to the order of presentation.

Another limitation with our current study is that the orientation of the displays differed for the two tasks. The emotional chimeric stimuli involved vertical displays of the faces, while the identity chimeric task involved displays with both vertical and horizontal aspects within the same stimuli, resulting in differences in orientation and complexity. The increased complexity and resulting visual scanning required for the identity task could have contributed to the results, as increased complexity with facial stimuli is thought to affect performance in ASD (Baron-Cohen, 2003; Teunisse \& de Gelder, 2003).

Although the conditions in the experiments were consistent for the two groups, we did not take any measurements involving visual path scanning. Therefore, we cannot determine if our results were influenced by differences between the groups on 
visual scanning or attention. Visual scanning paths for social stimuli are known to differ between controls and people with ASD (Klin, Jones, Schultz, Volkmar \& Cohen, 2002). The groups did not differ in RT to the stimuli, suggesting attention did not differ greatly between the two groups. However RTs do not give us a qualitative understanding about how the participants in our tasks were viewing the pictures or what aspects they were focusing on. Work is under way in our lab to investigate attention and scan paths during chimeric face judgments using gaze-tracking paradigms.

We included left-handers in each group, which may have an effect on the lateralisation of cognitive processing. The scores for the left-handed participants were consistent with the group averages for each task, and since the control participants showed the normal LVF bias consistent with previous findings, it is unlikely that handedness had an effect in our study. A recent study specifically investigating the effects of sex and handedness on the performance in chimeric face tasks showed no effect of handedness on the laterality scores in an emotional expression task (Rodway, Wright \& Hardie, 2003).

In conclusion, it is worth stating that since the paradigms used in this study are simple and non-verbal, they lend themselves to investigating hemispheric processing during social tasks in AS independent of executive function, even in people with limited language skills. In the present study, we have revealed a degree of normal face processing in AS in relation to emotion-perception (that is, a normal LVF bias), but a reduced effect compared to the controls during identity perception. This might reflect the under-development of a specialised fusiform face area reported in some (but not all) studies (Schultz et al., 2000; Pierce et al., 2001). Finally, we have revealed an anomalous LVF bias during processing of non-social stimuli, in people with AS, which may reflect an over-dependence on the right hemisphere. Whether this is because of a left-hemisphere dysfunction, or a right hemisphere over growth, would be an interesting question for future research. The findings are consistent with the Geschwind and Gallaburda (1987) model and may be evidence for an extreme right hemisphere style of processing in AS, which is consistent with being hyper-masculinized in neurocognitive terms. Functional imaging methods could compliment the neuropsychological methods reported here to test this extreme right hemisphere pattern further.

\section{ACKNOWLEDGMENTS}

The authors were supported by the Medical Research Council (MRC) UK and the National Alliance for Autism Research (NAAR) during the period of this work. We thank Hubert Jackson for creating the response box used to record responses, and we are grateful to the volunteers with Asperger syndrome who took part for their generous cooperation.

\section{REFERENCES}

Adolphs, R., Sears, L., \& Piven, J. (2000). Abnormal processing of social information from faces in autism. Journal of Cognitive Neuroscience, 13(2), 232-240.

Adolphs, R., Damasio, H., Tranel, D., Cooper, G., \& Damasio, A. R. (2000). A role for somatosensory cortices in the visual recognition of emotion as revealed by three-dimensional lesion mapping. Journal Neuroscience, 20(7), 2683-2690.

Adolphs, R., Jansari, A., \& Tranel, D. (2001). Hemispheric perception of emotional valence from facial expressions. Neuropsychology, 15(4), 516-524.

Allison, T., Puce, A., \& McCarthy, G. (2000). Social perception from visual cues: role of the STS region. Trends in Cognitiva Science, 4(7), 267-278.

APA (1994). DSM-IV diagnostic and statistical manual of mental disorders 4th ed. Washington DC.

Asperger, H. (1944) Die "Autistischen Psychopathen" im Kindesalter. Archiv fur Psychiatrie und Nervenkrankheiten, 117, 76136.

Baron-Cohen, S. (1988) Social and pragmatic deficits in autism: cognitive or affective? . Journal of Autism and Developmental Disorders, 18(3), 379-402.

Baron-Cohen, S. (1995). Mindblindness: an essay on autism and theory of mind. MIT Press/Bradford Books: Boston.

Baron-Cohen, S. (2003). The essential difference: Men, women and the extreme male brain. Penguin books: London.

Baron-Cohen, S., Spitz, A., \& Cross, P. (1993). Can children with autism recognize surprise? Cognition and Emotion, 7, 507-516.

Baron-Cohen, S., Wheelwright, S., \& Jolliffe, T. (1997). Is there a "language of the eyes"? Evidence from normal adults and adults with autism or Asperger syndrome. Visual Cognition, 4, 311-331.

Baron-Cohen, S., Wheelwright, S., Stone, V., \& Rutherford, M. (1999). A mathematician, a physicist, and a computer scientist with Asperger Syndrome: performance on folk psychology and folk physics test. Neurocase, 5, 475-483.

Baron-Cohen, S., Wheelwright, S., Skinner, R., Martin, J., \& Clubley, E. (2001). The autism-spectrum quotient (AQ): evidence from Asperger syndrome/high- functioning autism, males and females, scientists and mathematicians. Journal of Autism and Developement Disorders, 31(1), 5-17.

Blackstock, E. (1978) Cerebral asymmetry and the development of early infantile autism. Journal of Autism and Childhood Schizophrenia, 8, 339-353.

Bonda, E., Petrides, M., Ostry, D., \& Evans, A. (1996). Specific involvement of human parietal systems and the amygdala in the perception of biological motion. Journal of Neuroscience, $15,3737-3744$.

Bornstein, B. (1963). Prosopagnosia. In L. Halpern (Ed.), Problems of dynamic neurology. Hadassah Medical School: Jerusalem. 
Borod, J. C., Cicero, B. A., Obler, L. K., Welkowitz, J., Erhan, H. M., Santschi, C., Grunwald, I. S., Agosti, R. M., \& Whalen, J. R. (1998). Right hemisphere emotional perception: Evidence across multiple channels. Neuropsychology, 12(3), 446-458.

Boucher, J., \& Lewis, V. (1992). Unfamiliar face recognition in relatively able autistic children. Journal of Child Psychology Psychiatry, 33(5), 843-859.

Boucher, J., Lewis, V., \& Collis, G. (1998). Familiar face and voice matching and recognition in children with autism. Journal of Child Psychology and Psychiatry, 39(2), 171-181.

Bowers, D., Bauer, R. M., Coslett, H. B., \& Heilman, K. M. (1985). Processing of faces by patients with unilateral hemisphere lesions. Brain and Cognition, 4, 258-272.

Bowers, D., Bauer, R. M., \& Heilman, K. M. (1993). The nonverbal affect lexicon: Theoretical perspectives from neuropschological studies of affect perception. Neuropsychology, 7(4), 433-444.

Broca, M. P. (1865) Sur la siege de la faculté langage articulé. Bulletin of the Society of Anthropology, 6, 377-396.

Bruce, V., \& Young, A. (1986). Understanding face-recognition. British Journal of Psychology, 77, 305-327.

Bryson, S. E. (1990). Autism and anomolous handedness. In S. Coren (Ed.), Left handedness. Elsevier: Amsterdam.

Burt, M. D., \& Perrett, D. I. (1996). Perceptual asymmetries in judgements of facial attractiveness, age, gender, speech and expression. Neuropsychologia, 35(5), 685-693.

Campbell, R. (1978) Asymmetries in interpreting and expressing a posed facial expression. Cortex, 14, 327-342.

Celani, G., Battacchi, M. W., \& Arcidiacono, L. (1999). The understanding of the emotional meaning of facial expressions in people with autism. Journal of Autism and Development Disorders, 29(1), 57-66.

Christman, S. D., \& Hackworth, M. D. (1993). Equivalent perceptual asymmetries for free-viewing of positive and negative emotional expressions in chimeric faces. Neuropsychologia, 31, 621-624.

Cornish, K. M., \& McManus, I. C. (1996). Hand preference and hand skill in children with autism. Journal of Autism and Developmental Disorders, 26, 597-609.

Milner, B. (2002). Do I know you? Face perception and memory in patients with selective amygdalo-hippocampectomy. Neuropsychologia, 40(5), 530-538.

Davidson, R. J., \& Hugdahl, K. (1995). Brain asymmetry. MIT Press: Cambridge.

Davidson, R. J., Mednick, D., Moss, E., Saron, C., \& Schaffer, C. E. (1987). Ratings of emotion in faces are influenced by the visual field to which stimuli are presented. Brain and Cognition, 6(4), 403-411.

Davies, S., Bishop, D., Manstead, A. S., \& Tantam, D. (1994). Face perception in children with autism and Asperger's syndrome. Journal of Child Psychology and Psychiatry, 35(6), 1033-1057.

De Gelder, B., Vroomen, J., \& Van der Heide, L. (1991). Face recognition and lip-reading in autism. European Journal of Cognitive Psychology, 3, 69-86.

Drebing, C. E., Federman, E. J., Edington, P., \& Terzian, M. A. (1997). Affect identification bias demonstrated with individual chimeric faces. Perceptual and Motor Skills, 85, 1099-1104.

Ellis, H. D., Ellis, D. M., Fraser, W., \& Deb, S. (1994). A preliminary study of right hemisphere cognitive deficits and impaired social judgments among young people with Asperger syndrome. European Child and Adolescent Psychiatry, 3(4), 255-266.

Ellis, H. D., \& Gunter, H. L. (1999). Asperger syndrome: a simple matter of white matter? Trends in Cognitive Science, 3(5), 192200.

Escalante-Mead, P. R., Minshew, N., \& Sweeney, J. A. (2003). Abnormal brain lateralization in high-functioning autism. Journal of Autism and Developmental Disorders, 33(5), 539-543.

Fein, D., Waterhouse, L., Lucci, D., Pennington, B., \& Humes, M. (1985). Handedness and cognitve functions in pervasive developmental disorders. Journal of Autism and Developmental Disorders, 15, 323-333.

Fine, C., Lumsden, J., \& Blair, R. J. (2001). Dissociation between 'theory of mind' and executive functions in a patient with early left amygdala damage. Brain, 124(2), 287-298.

Forster, K. L., \& Forster, J. C. (2003). DMDX: a windows display program with millisecond accuracy. Behavior Research Methods Instruments Computers, 35(1), 116-124.

Geschwind, N., \& Galaburda, A. (1987). Cerebral lateralization. MA: MIT Press: Cambridge.

Gilbert, C., \& Bakan, P. (1973). Visual asymmetry in perception of faces. Neuropsychologia, 11, 355-362.

Gillberg, C. a. C. M. (2000). The Biology of the autistic syndromes. Cambridge University Press: Cambridge.

Golan O., Baron-Cohen S., \& Hill J. J. (in press). The Cambridge Mindreading (CAM) Face-Voice Battery: testing complex emotion recognition in adults with and without Asperger Syndrome. Journal of Autism and Developmental Disorders.

Grossman, J. B., Klin, A., Carter, A. S., \& Volkmar, F. R. (2000). Verbal bias in recognition of facial emotions in children with Asperger syndrome. Journal of Child Psychology and Psychiatry and Allied Disciplines, 41, 369-379.

Gunter, H. L., Ghaziuddin, M., \& Ellis, H. D. (2002). Asperger syndrome: tests of right hemisphere functioning and interhemispheric communication. Journal of Autism and Development Disorders, 32(4), 263-281.

Hauck, M., Fein, D., Maltby, N., Waterhouse, L., \& Feinstein, C. (1998). Memory for faces in children with autism. Child Neuropsychology, 4, 187-198.

Hauser, S., DeLong, G., \& Rosman, N. (1975). Pneumographic findings in the infantile autism syndrome. Brain, 98, 667-688.

Haxby, J. V., Ungerleider, L. G., Clark, V. P., Schouten, J. L., Hoffman, E. A., \& Martin, A. (1999). The effect of face inversion on activity in human neural systems for face and object perception. Neuron, 22(1), 189-199.

Haxby, J. V., Hoffman, E. A., \& Gobbini, M. I. (2000). The distributed human neural system for face perception. Trends in Cognitive Science, 4(6), 223-233.

Haxby, J. V., Hoffman, E. A., \& Gobbini, M. I. (2002). Human neural systems for face recognition and social communication. Biology Psychiatry, 51(1), 59-67.

Heller, W., \& Levy, J. (1981). Perception and expression of emotion in right-handers and left-handers. Neuropsychologia, 19(2), 263-272.

Hellige, J. B. (1993). Hemispheric asymmetry: What's right and what's left? Harvard University Press: Cambridge.

Hobson R. P. (1993). Autism and the development of mind: Lawrence Erlbaum Associates: Hillsdale, NJ.

Hoffman, E. A., \& Haxby, J. V. (2000). Distinct representations of eye gaze and identity in the distributed human neural system for face perception. Nature Neuroscience, 3(1), 80-84.

Hoptman Levy, M. J. J. (1988) Perceptual asymmetries in left- and right-handers for cartoon and real faces. Brain and Cognition, $8,178-188$.

Jackson, J. H. (1915) On the nature of duality of the brain. Brain, $38,80-103$

Jansari, A., Tranel, D., \& Adolphs, R. (2000). A valence-specific lateral bias for discriminating emotional facial expressions in free field. Cognition and Emotion, 14(3), 341-353.

Kanner, L. (1943) Autistic disturbance of affective contact. Nervous Child, 2, 217-250.

Kanwisher, N. (2000) Domain specificity in face perception. Nature Neuroscience, 3(8), 759-763.

Kanwisher, N., McDermott, J., \& Chun, M. M. (1997). The fusiform face area: a module in human extrastriate cortex specialized for face perception. Journal of Neuroscience, 17(11), 4302-4311.

Klin, A., Volkmar, F., Sparrow, S., Cicchetti, D., \& Rourke, B. (1995). Validity and neuropsychological characterization of Asperger syndrome: convergence with nonverbal learning 
disabilities syndrome. Journal of Child Psychology and Psychiatry, 36, 1127-1140.

Klin, A., Sparrow, S. S., de Bildt, A., Cicchetti, D. V., Cohen, D. J., \& Volkmar, F. R. (1999). A normed study of face recognition in autism and related disorders. Journal of Autism and Developmental Disorders, 29(6), 499-508.

Klin, A., Jones, W., Schultz, R., Volkmar, F., \& Cohen, D. (2002). Visual fixation patterns during viewing of naturalistic social situations as predictors of social competence in individuals with autism. Archives in General Psychiatry, 59(9), 809-816.

Kolb, B., Milner, B., \& Taylor, L. (1983). Perception of faces by patients with localized cortical excisions. Canadian Journal of Psychology, 37(1), 8-18.

Kurucz, J., \& Feldmar, G. (1979). Prosop-affective agnosia as a symptom of cerebral organic disease. Journal of the American Geriatrics Society, 27, 225-230.

Kurucz, J., Feldmar, G., \& Werner, W. (1979). Prosopo-affective agnosia associated with chronic organic brain syndrome. Journal of the American Geriatrics Society, 27, 91-95.

Langdell, T. (1978) Recognition of faces: an approach to the study of autism. Journal of Child Psychology and Psychiatry, 19, 225-238.

Levy, J., Heller, W., Banich, M. T., \& Burton, L. A. (1983). Asymmetry of perception in free viewing of chimeric faces. Brain and Cognition, 2(4), 404-419.

Lewin, J., Kohen, D., \& Mathew, G. (1993). Handedness in mental handicap: investigation into populations of Down's syndrome, epilepsy and autism. British Journal of Psychiatry, 163, 674 676.

Loveland, K. A., Tunali-Kotoski, B., Chen, Y. R., Ortegon, J., Pearson, D. A., Brelsford, K. A., \& Gibbs, M. C. (1997). Emotion recognition in autism: verbal and non-verbal information. Development and Psychopathology, 9, 579-593.

Luh, K. E., Ruekert, L. M., \& Levy, J. (1991). Perceptual asymmetries for free viewing of several types of chimeric stimuli. Brain and Cognition, 16, 83-103.

Lundqvist, D., Flykt, A., \& Ohman, A. (1998). The Karolinska Directed Emotional Faces-KDEF, CD ROM from the Department of Clinical Neuroscience, Psychology Section Karoinska Institute Stockholm. ISBN 91-630-71649.

Martin, I., \& McDonald, S. (2003). Weak coherence, no theory of mind, or executive dysfunction? Solving the puzzle of pragmatic language disorders. Brain and Language, 85(3), 451-466.

McCarthy, G., Puce, A., Gore, J. C., \& Allison, T. (1997). Facespecific processing in the human fusiform gyrus. Journal of Cognitive Neuroscience, 9, 605-610.

McManus, I. C., Murray, B., Doyle, K., \& Baron-Cohen, S. (1992). Handedness in childhood autism shows a dissociation of skill and preference. Cortex, 28, 373-381.

Milner, B., Taylor, L., \& Sperry, R. W. (1968). Lateralized suppression of dichotically presented digits after commissural section in man. Science, 161(837), 184-186.

Ozonoff, S., \& Miller, J. N. (1996). An exploration of right-hemisphere contributions to the pragmatic impairments of autism. Brain and Language, 52, 411-434.

Ozonoff, S., Pennington, B., \& Rogers, (1990). Are there emotion perception deficits in young autistic children? Journal of Child Psychology and Psychiatry, 31, 343-363.

Patterson, A., \& Zangwill, O. L (1944). Visual spatial agnosia associated with lesions of the right cerebral hemisphere. Brain, 61, 331-358.

Pierce, K., Muller, R. A., Ambrose, J., Allen, G., \& Courchesne, E. (2001). Face processing occurs outside the fusiform 'face area' in autism: Evidence from functional MRI. Brain, 124(10), 2059-2073.

Prior, M., \& Bradshaw, J. (1979). Hemispheric functioning in autistic children. Cortex, 15, 73-81.

Reuter-Lorenz, P., \& Davidson, R. J. (1981). Differential contributions of the two cerebral hemispheres to the percep- tion of happy and sad faces. Neuropsychologia, 19(4), 609613.

Reuter-Lorenz, P. A., Givis, R. P., \& Moscovitch, M. (1983). Hemispheric specialization and the perception of emotion: evidence from right-handers and from inverted and non-inverted left-handers. Neuropsychologia, 21(6), 687-692.

Riddoch, G. (1935) Visual disorientation in homonymous halffields. Brain, 58, 376-382.

Rinehart, N. J., Bradshaw, J. L., Brereton, A. V., \& Tonge, B. J. (2002a). A clinical and neurobehavioural review of highfunctioning autism and Asperger's disorder. Aust New Zealand Journal of Psychiatry, 36(6), 762-770.

Rinehart, N. J., Bradshaw, J. L., Brereton, A. V., \& Tonge, B. J. (2002b). Lateralization in individuals with high-functioning autism and Asperger's disorder: a frontostriatal model. Journal of Autism and Development Disorders, 32(4), 321-331.

Rodway, P., Wright, L., \& Hardie, S. (2003). The valence-specific laterality effect in free viewing conditions: The influence of sex, handedness, and response bias. Brain and Cognition, 53(3), 452-463.

Rossion, B., Dricot, L., Devolder, A., Bodart, J. M., Crommelinck, M., De Gelder, B., \& Zoontjes, R. (2000). Hemispheric asymmetries for whole-based and part-based face processing in the human fusiform gyrus. $J$ Cogn Neurosci, 12(5), 793-802.

Schultz, R. T., Gauthier, I., Klin, A., Fulbright, R. K., Anderson, A. W., Volkmar, F., Skudlarski, P., LaCadie, C., Cohen, D. J., \& Gore, J. C. (2000). Abnormal ventral temporal cortical activity during face discrimination among individuals with autism and Asperger syndrome. Archives of General Psychiatry, 57(4), 331-340.

Schultz, R. T., Romanski, L. M., \& Tsatsanis, K. D. (2000). Neurofunctional models of Autistic Disorder and Asperger Syndrome. In A. Klin, F. R. Volkmar \& S. S. Sparrow (Eds.), Asperger syndrome. (pp. 172-209). Guilford Press: New York.

Sperry, R. W., Gazzaniga, M. S., \& Bogen, J. E. (1969). Interhemispheric relationships: The neocortical commisures; Syndromes of hemisphere disconnection. In P. J. Vinken \& G. W. Bruyn (Eds.), Handbook of Clinical Neurology. Amsterdam: North-Holland.

Tanguay, P., Ornitz, E., Forsythe, A., \& Ritvo, E. (1976). Rapid eye movement (REM) activity in normal and autistic children during REM sleep. Journal of Autism and Developmental Disorders, 6(3), 275-287.

Tantam, D., Monaghan, L., Nicholson, H., \& Stirling, J. (1989). Autistic children's ability to interpret faces: a research note. Journal of Child Psychology and Psychiatry, 30, 623-630.

Teunisse, J. P., \& de Gelder, B. (2003). Face processing in adolescents with autistic disorder: the inversion and composite effects. Brain and Cognition, 52(3), 285-294.

Tranel, D., Damasio, A. R., \& Damasio, H. (1988). Intact recognition of facial expression, gender, and age in patients with impaired recognition of face identity. Neurology, 38(5), 690 696.

van Honk, J., Tuiten, A., van de Hout, M., Koppeschaar, H., Thijssen, J., de Haan, E., \& Verbaten, R. (1998). Baseline salivary cortisol levels and preconscious selective attention for threat. Psychoneuroendocrinology, 23, 741-747.

van Honk, J., Tuiten, A., van de Hout, M., Koppeschaar, H., Thijssen, J., de Haan, E., \& Verbaten, R. (2000). Conscious and preconscious selective attention to social threat: Different neuroendocrine response patterns. Psychoneuroendocrinology, $25,577-591$.

Wang, A. T., Dapretto, M., Hariri, A. R., Sigman, M., \& Bookheimer, S. Y. (2004). Neural correlates of facial affect processing in children and adolescents with autism spectrum disorder. Journal of the American Academy of Child and Adolescent Psychiatry, 43(4), 481-490. 
Waterhouse, L., Fein, D., \& Modahl, C. (1996). Neurofunctional mechanisms in autism. Psychological Review, 103, 457-489.

Wechsler, D. (1999). Wechsler abbreviated scale of intelligence. The Psychological Corporation: San Antonio.

Wernicke, C. (1874). Der aphasische Symptomencomplex. M. Cohn and Weigart. Breslau: Poland.
Williams, M. G. J., Mathews, A., \& Macleod, C. (1996). The emotional stroop task and psychopathology. Psychological Bulletin, 120(1), 3-24.

Wolff, W. (1933) The experimental study of forms of expression. Character and Personality, 2, 168-176.

Young, A. W. (1998). Face and mind. Oxford University Press: Oxford. 
Copyright of Journal of Autism \& Developmental Disorders is the property of Kluwer Academic Publishing and its content may not be copied or emailed to multiple sites or posted to a listserv without the copyright holder's express written permission. However, users may print, download, or email articles for individual use. 